Revue d'histoire de l'enfance « irrégulière »

Le Temps de l'histoire

Hors-série | 2001

Histoire et justice, panorama de la recherche

\title{
Le recours aux monitoires ecclésiastiques dans le Centre-Ouest français au siècle des Lumières
}

Fabrice Vigier

\section{(2) OpenEdition \\ Journals}

Édition électronique

URL : http://journals.openedition.org/rhei/459

DOI : 10.4000/rhei.459

ISBN : 978-2-7535-1641-0

ISSN : $1777-540 \mathrm{X}$

Éditeur

Presses universitaires de Rennes

Édition imprimée

Date de publication : 15 novembre 2001

Pagination : 221-239

ISSN : 1287-2431

Référence électronique

Fabrice Vigier, «Le recours aux monitoires ecclésiastiques dans le Centre-Ouest français au siècle des Lumières », Revue d'histoire de l'enfance «irrégulière » [En ligne], Hors-série | 2001, mis en ligne le 31 mai 2007, consulté le 19 avril 2019. URL : http://journals.openedition.org/rhei/459; DOI : 10.4000/rhei.459 


\section{Le recours aux monitoires}

\section{ecclésiastiques dans le Centre-Ouest}

\section{français au siècle des Lumières}

\section{(I'exemple du diocèse de Poitiers)}

Le monitoire ecclésiastique fait partie du "paysage" judiciaire de la France d'avant la Révolution. ${ }^{(2)}$ Il consiste en une sorte d'appel à témoins à propos d'un crime ou un délit récemment commis, que les desservants de paroisse ont obligation de lire plusieurs dimanches de suite au prône de la messe, en précisant bien à leurs fidèles qu'ils doivent absolument révéler ce qu'ils savent sur l'affaire sous peine d'excommunication. En d'autres termes, c'est une exhortation officielle à révélations que l'officiant profere publiquement avec menace d'exclusion automatique de la communauté catholique. A l'origine, cette pratique - qui remonte au XIIème siècle - relevait exclusivement du pouvoir clérical. Cependant, assez rapidement, les juridictions lä̈ques ont compris l'intérêt qu'elles pouvaient tirer de ce genre de procédure. C'est pourquoi elles s'en servent très largement dès le XVIème siècle, tandis que la grande ordonnance criminelle de 1670 en réglemente strictement l'utilisation dans ses articles organiques. ${ }^{(3)}$

Malgré ces spécificités qui font de cet usage un point de rencontre privilégié du "judiciaire" et du "sacré", le recours aux monitoires ecclésiastiques a été peu étudié jusqu'à présent. ${ }^{(4)}$ Cette étonnante carence a, semble-t-il, une double explication : elle est due à la fois à la relative rareté de ce genre de document ainsi qu'à son grand éparpillement dans les fonds d'archives français. En dépit de cette difficulté, on a pu retrouver la trace d'un nombre assez significatif de ces monitoires, tous promulgués dans le diocèse de Poitiers entre 1670 et la Révolution. On les a dénichés dans trois dépôts d'archives bien distincts, en l'occurrence dans les registres de délibérations du chapitre Saint-Hilaire le Grand de Poitiers (37 actes), ${ }^{(5)}$ dans les minutes de deux juridictions poitevines
Fabrice

Vigier $^{(1)}$
(1) Historien, maître

de conférences

d'Histoire moderne, Gerhico.

(2) Plusieurs personnes m’ont apporté leur concours, au cours de cette enquête, en me donnant d'utiles indications archivistiques ou bibliographiques. Je tiens, en particulier, à remercier Vanessa Audebert, Jean-Marie Augustin, Christelle Bigot, Virginie Blin, Edouard Bouyé, Frédéric Chauvaud, Yves

Couturier, Robert Favreau, Dominique Guillemet, Valérie Hervouet, Christelle Lebellec, Soline Lelot, Nicole Pellegrin et Jacques Péret.

(3) Raoul NAZ, article

Fabrice Vigier / pp. 221 à 239 
"Monitoire", dans

Dictionnaire de droit canonique (sous la direction de Raoul NAZ), Paris, 1957, Tome VI, colonnes 940-942.

(4) La seule synthèse existant sur le sujet demeure l'ouvrage ancien de Pierre DUPONT, Les monitoires à fin de révélations dans la procédure séculière, Paris, 1930.

(5) Archives départementales de la Vienne (ADV), G 547 à G 570.

(6) Il s'agit, en l'occurrence des minutes de la justice seigneuriale de Saint-Hilaire le Grand de Poitiers [Archives départementales de la Vienne, G 654 à 674 (1701-1788)], et de celles de la sénéchaussée et siège présidial de Poitiers [ADV, B I/2/6 à B I/2/40 (1700-1740), B I/2/56 à B I/2/80 (17461755), B I/2/89 à B I/2/116 (1759-1770), B $\mathrm{I} / 2 / 157$ à $\mathrm{B} \mathrm{I} / 2 / 180$ bis (1780-1784)].
(9 actes) ${ }^{(6)}$ et dans trois petits "recueils" de documents de ce type (15 actes) déposés aux Archives départementales de la Vienne. ${ }^{(7)}$ A cette collecte archivistique, on a pris le parti d'ajouter les deux procédures de ce genre citées dans la thèse classique de Marie-Louise Fracard intitulée La fin de l'Ancien Régime à Niort. Essai de sociologie religieuse parue en $1956{ }^{(8)} \mathrm{Au}$ total, ce dépouillement a permis de réunir une collection de 57 monitoires différents, parfois accompagnés (dans 9 cas) de papiers supplétifs concernant l'affaire.

Même si l'échantillon n'est peut-être pas aussi important qu'on aurait pu l'espérer, il offre néanmoins l'opportunité d'avoir une petite idée sur ce genre d'actions en justice. Il donne, en particulier, la possibilité d'appréhender concrètement la procédure suivie, de cerner les types d'affaires et de délits pour lesquels on promulgue un monitoire, et enfin de juger de l'utilité et des éventuels défauts de ces appels à témoins dans le Poitou de la fin du XVIIème et du XVIIIème siècle.

\section{De la procédure suivie}

Malgré leurs lacunes, les documents consultés permettent de connaître la procédure suivie. Celle-ci suit, en fait, scrupuleusement les prescriptions de l'ordonnance criminelle de $1670 .^{(9)}$ Elle fait intervenir plusieurs "acteurs" différents et se déroule effectivement en trois temps.

\section{La requête d'une autorité judiciaire}

Tout d'abord, toute action en justice de ce genre commence par une requête d'une autorité judiciaire. Toute juridiction d'Ancien Régime a, en effet, la possibilité de demander la promulgation d'un monitoire, si le délit (ou le crime) est grave et si aucun élément tangible ne permet de le résoudre.

Dans l'échantillon de 49 affaires pour lesquelles on dispose de l'information, seulement six institutions de justice différentes ont recouru à ce type de procédure. Les deux juridictions poitevines s'y taillent la part du lion. La "sénéchaussée et siège présidial" de Poitiers est ainsi à l'origine de pas moins de 39 monitoires (soit 79,60\% du total), et devance très largement le tribunal seigneurial du bourg de Saint-Hilaire 
Le Grand et ses 5 requêtes (10,20 \% de l'ensemble). Ce poids écrasant des organes de justice de la cité pictave n'est guère étonnant : il s'explique à la fois par la nature du corpus considéré (essentiellement de la ville même de Poitiers), et par l'importance du ressort de la sénéchaussée de Poitiers qui couvre près de la moitié du territoire du diocèse. En revanche, le fait de relever trois organes parisiens dans cet échantillon peut, de prime abord, surprendre. Il s'agit, en l'occurrence, du Parlement de Paris (1 monitoire) et de deux juridictions établies exceptionnellement - et provisoirement - par le pouvoir royal pour régler certains problèmes financiers en 1688 et 1716 ( 3 monitoires). Même si elle est numériquement marginale, leur présence montre toutefois le caractère parfois national de ce genre de procédure : elle prouve que toutes les autorités judiciaires françaises - et non seulement poitevines - peuvent engager ce genre de procédure dans le diocèse de Poitiers pour avoir des éclaircissements sur une affaire importante. Enfin, signalons qu'un dernier monitoire n'émane pas, à proprement parler, d'une juridiction, mais de l'intendant du Poitou (Nicolas Étienne Roujault) qui porte néanmoins le titre officiel d'" intendant de justice, police et finances".

Quoi qu'il en soit (et d'où qu'elles viennent), toutes ces demandes d'actions judiciaires sont adressées à l'officialité diocésaine : c'est le deuxième temps de la procédure.

\section{La saisie de l'official du diocèse}

Pour être exécutoires, ces demandes d'appels à témoins doivent être dûment acceptées par l'official du diocèse. Cependant, ce dernier n'a pas vraiment le choix. L'ordonnance criminelle de 1670 précise, en effet, que le juge d'Église a l'obligation d'accorder et de publier le monitoire sous "peine de saisie de [son] temporel " par une juridiction royale. ${ }^{(10)}$ Malgré les protestations répétées de l'Assemblée générale du Clergé de France, l'officialité diocésaine n'est donc, dans ces conditions, qu'une simple chambre d'enregistrement. Cela se perçoit d'ailleurs assez bien dans nos documents, puisqu'il n'existe généralement aucun délai entre la date de la requête et celle d'impression du monitoire. Pour ne prendre que ces exemples, l'official Sébastien Le Carlier enregistre le jour même
(7) ADV, GG 49

(monitoires) ; G

Supplément 6 (officialité de Poitiers) ; J 1101

(monitoires 1702-1747).

(8) Marie-Louise FRA-

CARD, La fin de l'Ancien

Régime à Niort. Essai de

sociologie religieuse, Paris,

1956, pp. 79-80.

(9) André LAINGUI

et Arlette LEBIGRE,

Histoire du droit pénal,

Tome II, La procédure criminelle, Paris, 1980, p. 92 .

(10) JOURDAN,

DECRUSY, ISAM-

BERT, Recueil général des anciennes lois françaises depuis l'an 420 jusqu'à la Révolution de 1789, Tome XVIII, 1661-1671, Paris, 1821-1833, p. 384. 
(11) ADV, B I/2/58

(monitoire du 13 juin 1747) ; B I/2/67 (monitoire du 13 juin 1750).

(12) ADV, B I/2/104 (monitoire du 17 janvier 1765) ; G 673 (monitoire du 14 février 1781).

(13) ADV, B I/2/58 (monitoire du 29 avril 1747) ; B I/2/76 (monitoire du 1er juillet 1755) ; B I/2/180 bis (monitoire du 19 mars 1784).

(14) ADV, B I/2/67 (monitoire du 13 juin 1750). les demandes du "procureur $d u$ roi » au présidial de Poitiers du 29 avril

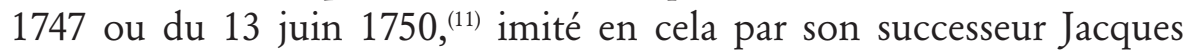
Louis Decressac qui agit aussi diligemment pour celles qu'on lui adresse le 17 janvier 1765 ou encore le 14 février 1781. ${ }^{(12)}$

Cependant, même s'il n'est pas chargé de décider du bien fondé de ce genre d'actions en justice, le rôle du premier dignitaire de l'officialité n'en reste pas moins essentiel. Sa responsabilité, en la circonstance, est triple :

- Tout d'abord, c'est à lui - et à lui seul - que revient la rude tâche de rédiger le texte officiel des monitoires. L'exercice est difficile et obéit à des règles bien strictes. D’une part, leur énoncé ne doit pas être trop long, de façon à pouvoir être éventuellement placardé sur les portes des églises du diocèse : ceux de notre corpus comprennent ainsi entre 15 et 26 lignes et tiennent tous sur une seule page. Il faut, d'autre part, que ces textes soient adaptés à l'usage qu'on désire en faire, tout en étant relativement structurés. C'est pourquoi ils adoptent tous, à peu de chose près, le même schéma et le même ton. Ils commencent généralement (dans les premières lignes de l'acte) par rappeler les règles de publication du monitoire, se poursuivent par le récit (le plus précis possible) de l'affaire incriminée, avant de se terminer par la demande d'appel à révélations sous peine de "censures ecclésiastiques » et d' excommunication".

- Une fois rédigés (et le plus souvent imprimés), l'official a également la mission d'envoyer ces monitoires aux membres du clergé paroissial. Le nombre de desservants à qui il adresse ces documents est très variable, et dépend aussi bien de la nature et de l'importance de l'affaire, que du lieu où elle s'est déroulée. Dans notre échantillon, et même si nos renseignements dans ce domaine sont loin d'être complets, on rencontre apparemment tous les cas de figure. Ainsi, au moins 21 monitoires de notre corpus sont publiés dans une seule paroisse du diocèse, tandis qu'au moins six autres sont visiblement envoyés à l'ensemble des responsables religieux de la circonscription. Entre ces deux "extrêmes", on sait par ailleurs que dix de nos appels à témoins sont promulgués par au moins trois curés, trois autres par au moins quatre desservants ${ }^{(13)}$ et un dernier par au moins dix-sept titulaires d'une paroisse poitevine. ${ }^{(14)}$ 
- Enfin, et à nouveau à la requête d'une autorité judiciaire, l'official est parfois amené à publier un reagrave. Ce genre d'action en justice ressemble, à s'y méprendre, à un monitoire dont il n'est d'ailleurs qu'une simple reprise. Le reagrave n'est effectivement qu'un nouvel appel à révélations (avec les mêmes menaces d'excommunication) sur un crime ou un délit pour lequel la promulgation d'un monitoire n'a pas eu l'effet escompté. Cette procédure, sans être exceptionnelle, n'est cependant pas très fréquente : sur les 57 actes de notre échantillon, 9 seulement sont suivis et prolongés d'un reagrave entre 1670 et 1789 . Quant à leurs dates de publication, elles ne sont pas toujours très rapides : ainsi, si 6 reagraves font presque immédiatement suite aux différentes annonces de monitoires, les 3 derniers sont proclamés plus de deux mois après les premiers appels à témoins.

L'official du diocèse joue donc un rôle essentiel dans ces diverses affaires : c'est son sceau et sa signature qui rendent crédibles les menaces d'exclusion de la communauté catholique. Toutefois, une fois ces tâches "techniques" accomplies (enregistrement des demandes, travail de rédaction et de diffusion), il n’intervient plus vraiment dans la procédure.

\section{La "fulmination" des responsables de paroisse}

Même s'ils n'interviennent qu'en troisième position, les membres du clergé paroissial sont incontestablement les personnages les plus importants dans ce genre d'action en justice. Ces derniers sont, en effet, les seuls habilités à recevoir les monitoires, mais sont expressément contraints selon les termes de l'ordonnance criminelle de 1670 - d'en faire la publication sous "peine de saisie de leur temporel ». La plupart du temps, c'est le curé de paroisse qui se charge de cette mission. Cependant, il arrive parfois qu'il en confie l'exécution à son suppléant : au moins trois monitoires de notre échantillon sont d'ailleurs annoncés de cette façon.

En tout cas, qu'ils soient simples vicaires ou curés, on attend alors d'eux qu'ils assument trois types de tâches :

- En premier lieu, ces responsables religieux ont l'obligation de faire lecture de ces monitoires "trois dimanches consécutifs" au "prône de la Messe Paroissiale ». Si l'on se fie aux rituels diocésains de 1712 et surtout 
(15) Bibliothèque

municipale de Poitiers (médiathèque François Mitterrand), DP 474, Rituale Romanum ad Usum Dioecesis

Pictaviensis, Pictavii, 1712, p. 562-565 ; Rituel du Diocèse de Poitiers, publié par l'autorité de Monseigneur Martial Louis De Beaupoil De Saint-Aulaire, évêque de Poitiers, Poitiers, 1766, Première Partie, pp. 270274.

(16) DURAND DE MAILLANE,

Dictionnaire de droit canonique et de pratique bénéficiale, Lyon, 1776, t. IV, article "Monitoire", p. 103.

(17) ADV, B I/2/76 (monitoire du 1er juillet 1755).

(18) Daniel JOUSSE, Traité du gouvernement spirituel et temporel des paroisses, Paris, 1769 , p. $306-307$. de 1766, ces clercs ne doivent pas se contenter d'en lire l'énoncé : il leur faut aussi, durant ces trois semaines, en faire le commentaire à leurs paroissiens, en insistant bien sur la gravité de la peine d'excommunication. ${ }^{(1)}$ L'objectif des prêtres en ces circonstances est clair : impressionner et faire suffisamment peur à leurs fidèles, pour que ceux qui savent quelque chose sur l'affaire viennent à révélations.

- Ces desservants sont justement les seuls autorisés à recevoir les éventuels témoignages de leurs concitoyens. ${ }^{(16)}$ La plupart du temps, l'opération se déroule au domicile de l'officiant et beaucoup plus rarement chez les déposants ( 2 cas rencontrés seulement sur un total de 351 "révélations»). Ceux qui désirent témoigner tiennent, par ailleurs, à ce que leur initiative demeure secrète : c'est pourquoi ils ne se précipitent pas dans la maison du desservant juste après l'annonce dominicale, mais attendent généralement plusieurs heures, voire plusieurs jours, pour venir dire ce qu'ils croient savoir sur l'affaire. Quant au nombre de visiteurs du curé en ces occasions, il s'avère très variable. Dans l'échantillon de 18 communautés d'habitants (villages, bourgs ou paroisses urbaines) pour lesquelles la publication d'un monitoire a suscité au moins une "révélation ", l'officiant concerné a ainsi auditionné un seul témoignage dans 6 paroisses, entre 2 et 6 dépositions dans 8 autres, et plus de 13 «révélations» dans les 4 dernières. Précisons que le « record" en la matière appartient au monitoire du 1er juillet 1755 dont la publication dans la petite bourgade de Pleumartin (en Châtelleraudais) entraine l'enregistrement de quelques 186 "révélations » par le desservant dans les premiers jours de l'été 1755 . $^{(17)}$

- Enfin, la dernière mission des curés (ou vicaires) consiste à consigner par écrit les paroles de leurs ouailles, et à les envoyer à la juridiction qui instruit l'affaire. ${ }^{(18)}$ Cette dernière - qu'il s'agisse du présidial de Poitiers ou du Parlement de Paris - a alors la possibilité de se "servir" du document rédigé par l'officiant pour convoquer de nouveaux témoins, et trouver les possibles responsables du crime ou du délit non résolus. D'ailleurs, dans au moins 8 affaires de notre corpus, l'appel à témoins prononcé par le desservant a débouché sur de nouvelles auditions et semble avoir permis d'arrêter des coupables. 
Par ces diverses obligations, les titulaires d'une cure jouent donc un rôle important - voire clef - dans ce genre de procédure. Toute action de ce type ne peut effectivement aboutir et s'avérer efficace sans la participation pleine et entière des officiants. Les autorités judiciaires du moment en ont d'ailleurs pleinement conscience puisqu'elles accordent au clergé paroissial poitevin quelques indemnités financières pour la publication de chaque nouveau monitoire. ${ }^{(19)}$

\section{Des affaires concernées}

Plus que de cerner la procédure suivie, les 57 actes retrouvés aux Archives départementales de la Vienne offrent surtout la possibilité de savoir pour quels types de crimes et de délits on promulgue un monitoire dans le diocèse de Poitiers entre 1670 et 1789. Sans en donner une liste exhaustive qui n'aurait guère d'intérêt, on peut se risquer - parfois arbitrairement - à les classer et à les regrouper en quatre grandes catégories d'affaires, tout en étant attentif aux catégories sociales des victimes des méfaits que dénoncent ces monitoires pictaves.

\section{Une grande majorité de vols et de "divertissements" de biens}

Les histoires de "vols" et de "divertissements " de biens arrivent assez largement en tête du corpus, avec pas moins de 35 monitoires (61,40\% du total). Loin d'être semblables et uniformes, ces délits ne sont cependant pas tous de la même nature :

- Avec pas moins de 16 documents de ce genre, les plus nombreux sont les vols qui interviennent au domicile d'une personne défunte ou sur le point de décéder. L'acte doit sembler particulièrement odieux et répréhensible aux autorités judiciaires du moment, puisque c'est - sans contestation possible - pour ce type d'exactions qu'elles réclament le plus de monitoires à l'official du diocèse $(28 \%$ environ de l'ensemble de l'échantillon). Ces opérations délictueuses concernent généralement les «menbles» ou les «vaisselles", mais aussi quelquefois les "bestiaux" ou l'«argenterie ». Elles représentent souvent un dommage important : on aurait ainsi volé de l'« or et argent monnayé " au domicile du défunt « $s$ [ieur] Binet " au mois d'avril $1689,{ }^{(20)}$ ainsi que chez «feu [...] Jacques
(19) Bibliothèque municipale de Poitiers, RC 188, Reiglement de Monseigneur l'Official de Poictiers touchant les salaires que doivent prendre les gens d'Église, pour les Convoy, Enterremens, Messes, Vigiles, Fiançailles, Mariages, Monitoires, \& autres droicts d'Église, Poitiers, 30 mars 1620, p. 5.

(20) ADV, G 552, délibération capitulaire de Saint-Hilaire Le Grand de Poitiers du dimanche 17 avril 1689, p. 90 (monitoire du 15 avril 1689). 
(21) ADV, G 557, délibération capitulaire de Saint-Hilaire Le Grand de Poitiers du samedi 10 août 1709 , p. 48 (monitoire du 10 août 1709).

(22) ADV, G 561, délibération capitulaire de Saint-Hilaire Le Grand de Poitiers du samedi 5 août 1719, p. 29 (monitoire du 1er juillet 1719).

(23) ADV, G 567, registre 154 , délibération capitulaire de SaintHilaire Le Grand de Poitiers du samedi 9 mars 1765, p. 292 (monitoire du 8 mars 1765).

(24) ADV, B I/2/89 (monitoire du 24 janvier 1759).

(25) ADV, G 551, délibération capitulaire de Saint-Hilaire Le Grand de Poitiers du samedi 17 mai 1687, p. 89 (monitoire du 15 mai 1687).

(26) ADV, G 557, délibération capitulaire de Saint-Hilaire Le Grand
Rousseau » en août 1709. (21) En certaines circonstances, ces "divertissements de biens " sont, par ailleurs, visiblement très organisés, comme celui qui a lieu chez Michel Métoys (de la paroisse de Saint-Pierre L'Hospitalier de Poitiers) alors mourant une nuit du mois de mars 1719, au cours de laquelle "plusieurs personnes " "ont enlevé nuitement [...] conduits et faits conduire en leurs maisons" plusieurs " meubles, coffres et au [tres] choses de valleur considérable ${ }^{(22)}$ ". Quant aux "victimes" (mortes !) de ces méfaits, elles semblent appartenir à des milieux sociaux relativement variés, puisqu'on y recense 6 ecclésiastiques, 6 artisans ou marchands, 2 veuves, un procureur de justice et un avocat.

- Beaucoup plus classiques, on trouve ensuite 11 monitoires dénonçant des effractions faites aux domiciles de particuliers "vivants " (soit près de $20 \%$ du total du corpus). Les biens dérobés sont, comme auparavant, de valeur très variable : il peut s'agir aussi bien de "meubles » ordinaires, de "morceaux de viande " et de poissons, ${ }^{(23)}$ que de sommes rondelettes comme celle de "deux mille sept cent quatre-vingt-une livres" que des quidams accaparent en l'"Abbaye royale de Notre-Dame de l'Étoile " entre les 6 et 7 août 1758. ${ }^{(24)}$ Cependant, en ce qui concerne les victimes de ces "divertissements", elles semblent ne pas avoir exactement le même profil que pour les vols précédents. On y rencontre effectivement une plus forte proportion de femmes (5 sur 11 cas), et de gens plus prestigieux socialement. Parmi eux, figurent ainsi Marthe Landru " femme non commune [...] de Pierre Pitton receveur général des finances " de la généralité de Poitiers, ${ }^{(25)}$ "Jean Labbé Ecuyer $S$ [eigneur] des Bordes ${ }^{(26)}$ ", ou encore "Claude Bardeau, Receveur des Décimes" du diocèse. ${ }^{(27)}$ Toutefois, la victime la plus illustre de cet échantillon est, sans aucun doute, " $\mathrm{Mr}$ De Beauregard [...] Subdélégué de Mr l'Intendant de cette province " au domicile duquel des inconnus "prirent \& emportèrent la somme de neuf cent livres » le soir de Noël $1749 .{ }^{(28)}$

- Dans le panel d'exactions recensées dans ces monitoires poitevins, quelques-uns (5 en tout, soit 8,77\% du total) ont trait à des "divertissements de biens " assez particuliers, puisqu'ils concernent des " titres et [autres] papiers". A vrai dire, ces vols sont assez spéciaux : ils ne sont pas toujours effectués avec effraction de domicile, et apparaissent sou- 
vent comme d'habiles et savantes subtilisations de documents. C'est la mésaventure qui advient, pour ne prendre que cet exemple, à Jean Descorolles qui s'est fait dérober "par finesse ou autrement " divers " Titres ¿ Papiers " au mois de juin 1691.(29) Quant aux documents perdus, volés ou égarés pour lesquels on engage ce genre de procédure, ils sont toujours importants : on y trouve deux "titres en féodalité " appartenant respectivement à "l'abbaye royalle de Ste Croix ${ }^{(30)}$ " et à l'évêché, ${ }^{(31)}$ un état des revenus du bénéfice de l' "Abbé de Saint Jean près Poictiers ${ }^{(32)}$ ", plusieurs " titres et papiers " de l'échevinage de Niort, ${ }^{(33)}$ ainsi qu'une " minutte d'obligation de deux mille cent onze livres" au nom de "Mr Abraham Corbin procureur au présidial(34) ".

- Pour ce qui est, enfin, des derniers vols pour lesquels les autorités judiciaires décident de faire publier un appel à témoins, ils s'avèrent beaucoup plus marginaux. On y découvre, tout d'abord, deux étonnantes effractions dans des domaines privés : la première dans plusieurs jardins du bourg de Saint-Hilaire de Poitiers où " certains libertins mal intentionnés " ont pris notamment "plusieurs myrtes [...] et 212 artichauts avec un cadran solaire " durant la nuit du 7 juin 1747,35 et la seconde à Cissé, dans la propriété du notaire Vincent Hilaire Du Tertre de la Coudre, où des inconnus ont " arraché dix-sept arbres ormeaux " dans la "nuit du dimanche jour des Rois " 1765. ${ }^{(36)}$ Quant au dernier " divertissement de biens " de notre corpus - si l'on peut le désigner ainsi -, il est encore plus surprenant : daté du printemps 1696, il demande en effet à avoir des éclaircissements sur "des sommes de deniers " trouvées accidentellement "par certains particuliers employés à curer et nettoyer lieux communs dans un logis qui appartient en partie [au] sieur Maisondieu ${ }^{(37) " .}$

En tout cas, ces histoires de vols sont, sans surprise, les plus nombreuses. Sur ce point, les monitoires diffusés par l'official de diocèse correspondent tout à fait à l'activité des principales juridictions poitevines chez qui les délits jugés de ce genre sont prépondérants au siècle des Lumières.

\section{Une minorité d'actes de violence}

Après les vols, les actes de violence arrivent en seconde position dans notre corpus, avec pas moins de 11 monitoires (soit près de $20 \%$ du total). de Poitiers du dimanche

7 septembre 1710 ,

p. 152 (monitoire du

6 septembre 1710).

(27) ADV, G 673

(monitoire du 14 février 1781) ; G 569, registre

156, délibération capitulaire de Saint-Hilaire Le Grand de Poitiers du samedi 10 mars 1781 , p. 119.

(28) ADV, B I/2/67 (monitoire du 23 janvier 1750).

(29) ADV, G Supplément 6 (monitoire du 8 juin 1691).

(30) ADV, G 552, délibération capitulaire de Saint-Hilaire Le Grand de Poitiers du dimanche 27 mars 1689 , p. 87 (monitoire du 24 mars 1689).

(31) ADV, J 1101 ; B $\mathrm{I} / 2 / 58$ (monitoire du 29 avril 1747).

(32) ADV, G Supplément 6 (monitoire du 8 juin 1691).

(33) Marie-Louise FRACARD, op. cit., p. 79 . 
(34) ADV, G 553, délibération capitulaire du dimanche 25 mai 1692 , p. 56 (monitoire du 23 mai 1692).

(35) ADV, J 1101 (monitoire du 13 juin 1747).

(36) ADV, B I/2/104 (monitoire du 17 janvier 1765).

(37) ADV, G 555, délibération capitulaire de Saint-Hilaire Le Grand de Poitiers du samedi 9 juin 1696, p. 121 (monitoire daté sans doute de fin mai ou début juin 1696).

(38) ADV, B I/2/76 (monitoire du 1er juillet 1755).

(39) ADV, G 551, délibération capitulaire de Saint-Hilaire Le Grand de Poitiers du dimanche 5 août 1685 , p. 30 (monitoire du 17 juillet 1685).

(40) ADV, G 551, délibération capitulaire de Saint-Hilaire Le Grand de Poitiers du samedi 23 février 1686 (moni-
Ces agressions "physiques " à l'encontre de particuliers, bien qu'elles soient importantes, ne sont cependant pas toutes de la même gravité.

En premier lieu, 6 de ces documents dénoncent des violences se terminant par mort d'homme. Pour 5 de ces faits tragiques, on ne sait absolument rien des circonstances des crimes, et les appels à témoins sont prononcés avant tout pour " avoir [des] preuves » et des "éclaircissements " sur les meurtres commis. En revanche, dans le monitoire du 1er juillet 1755, on apprend que la victime est morte après qu'on l'eut longuement torturée près d'un feu et "brullé tout le dos ${ }^{(38)}$ ». Si cruels soient-ils, ces assassinats sont perpétrés sur des individus de milieux sociaux assez variés. On y recense ainsi deux simples prêtres (Pierre Ancelin "vicaire servant [...] Ligugé et Smarve "(39) " et le "nommé Branlé clerc [...] de Saint-Porchaire de Poitiers ${ }^{(40)}$ "), un petit paysan ("Pierre Bombard, vivant Bordier [...] paroisse d'Anxaumont $\left.{ }^{(41)} »\right)$, un huissier de Châtellerault ${ }^{(42)}$ et un "commis aux Aides " de Poitiers. ${ }^{(43)}$ Mais on a aussi l'énorme surprise d'y découvrir une autorité provinciale de premier plan en la personne d' "Antoine Micheau Dumeslier Seigneur de la Leuf et du Sceau, avocat en parlement et Subdélégué de M. l'Intendant [...] au département de Montmorillon ", trouvé assassiné le 10 décembre 1783. ${ }^{(44)}$

Fort heureusement, les autres actes violents de notre petit échantillon ne se terminent pas de manière aussi dramatique. Ce sont cependant tous des délits graves et fort répréhensibles pour la justice d'Ancien Régime. D'ailleurs, il s'en est fallu visiblement de peu pour que deux d'entre eux ne débouchent sur de véritables meurtres. C'est le cas des agressions "musclées" dont sont respectivement victimes "René Victor Bodin seigneur de la Girauderie " "maltraité le 3 novembre [1715] [...] par cinq quidams sur les 4 à 5 beures du soir au village de Moulinet ${ }^{(45)}$ ", ainsi que le dénommé " $M$. de Breuillac " trouvé grièvement blessé dans les fossés de Niort le 10 avril 1738. ${ }^{(46)}$ Quant aux autres violences, elles s'avèrent moins conventionnelles, mais parfois plus sournoises. Ainsi, deux d'entre elles sont des tentatives d'empoisonnement contre des religieuses de Poitiers. La première aurait pu avoir pour victime "la Demoiselle Dupont, Pensionnaire au Couvent des dames Religieuses Hospitalières " à qui des inconnus auraient porté "en présent une bouteille de vin \& une boëte de 
confiture " dans lesquels ils auraient "répandu du poison " à la fin de l'année 1742, ${ }^{(47)}$ tandis que la seconde visait " la damoiselle Augier et sa fille de chambre, pensionnaires " de l'abbaye Sainte-Croix le 13 avril 1749.(48)

Toutefois, le monitoire qui relate l'action la plus spectaculaire est, sans contestation possible, celui qui est promulgué le 13 juin 1750 . Il y est question de l'évasion du bandit Jean Ouvrard " dit Lavarenne » alors qu'on le "conduisait [...] en les prisons de la conciergerie du Palais à Paris [...] dans la voiture publique ". On désire évidemment le retrouver, mais surtout mettre la main sur ses complices - «buit à dix particuliers masqués, habillés en redingottes ¿ armés " - qui auraient intercepté le convoi, menacé les gardes et libéré le prisonnier "sur le grand chemin de Châtellerault à Ingrande, la nuit du 4 au 5 [...] mars $1750 » .{ }^{(49)}$

\section{Plusieurs atteintes à l'Église catholique et aux prérogatives royales}

Numériquement aussi importants que ces actes de violence, on recense enfin 11 monitoires dénonçant des atteintes commises à l'encontre du catholicisme et des prérogatives du Roi. Plus que les vols et les assassinats, ces délits sont primordiaux car ils sont souvent très durement punis par les autorités judiciaires d'Ancien Régime.

Tout d'abord, pas moins de 6 documents de notre corpus font référence à des " attentats " visant - au sens large du terme - des lieux de culte catholique. Ceux-ci sont, à vrai dire, relativement variés. Il peut s'agir, en premier lieu, d'incidents marginaux, voire anecdotiques, comme l'appel à témoins qui entend découvrir " ceux qui ont proféré [...] plusieurs parolles insolentes [...] contre [...] les vitres " $\mathrm{du}$ couvent des "dames ursulines" de Poitiers au mois de juillet 1712..$^{(50)}$ Deux monitoires dénoncent, par ailleurs, des actes de vandalisme : il est ainsi question de "quelques particuliers pris de vin" qui "entreprirent de [...] démolir [...] au mépris de la religion une Croix de pierre " dans le cimetière Saint-Jacques de la cité pictave en octobre $1717,{ }^{(51)}$ ou encore de "gens mal intentionnés" qui auraient "jetté par terre la statue de la très Sainte Vierge et du Saint Enfant Jésus " dans une chapelle du bourg de Saint-Hilaire dans "la nuit du seize au dix-sept " août 1740. ${ }^{(52)}$ On rencontre, en outre, deux cas de vols : le premier a pour objet une "Image ou Figure de la Très Sainte Vierge [...] avec toire du 21 février 1686).

(41) ADV, G Supplément 6 (monitoire du 30 janvier 1783).

(42) ADV, B I/2/76 (monitoire du 1er juillet 1755).

(43) ADV, G 550, délibération capitulaire de Saint-Hilaire Le Grand de Poitiers du dimanche 29 octobre 1684, p. 91 (monitoire du 21 octobre 1684).

(44) ADV, B I/2/180 bis (monitoire du 19 mars 1784).

(45) ADV, G 559, délibération capitulaire de Saint-Hilaire Le Grand de Poitiers du samedi 11 janvier 1716 (monitoire du 27 décembre 1715).

(46) Marie-Louise FRACARD, op. cit., p. 79.

(47) ADV, J 1101 (monitoire du 4 décembre 1742).

(48) ADV, G 566, délibération capitulaire de Saint-Hilaire Le Grand de Poitiers du samedi 
14 juin 1749, pp. 127-

128 (monitoire du 9 mai 1749).

(49) ADV, B I/2/67 ;

GG 49 (monitoire du

13 juin 1750).

(50) ADV, G 557, délibération capitulaire de Saint-Hilaire Le Grand de Poitiers du dimanche 31 juillet 1712, p. 301 (monitoire du 28 juillet 1712).

(51) ADV, G 559, délibération capitulaire de Saint-Hilaire Le Grand de Poitiers du mardi 12 octobre 1717, p. 141 (monitoire du 10 octobre 1717).

(52) ADV, J 1101 (monitoire du 19 août 1740).

(53) ADV, G Supplément 6 (monitoire du 5 septembre 1682).

(54) ADV, G Supplément 6 (monitoire du 14 juillet 1780).

(55) ADV, G 558, délibération capitulaire de Saint-Hilaire Le Grand de Poitiers du mardi 27 novembre tous ses ornemens " dérobée dans l'église de l'abbaye de Saint-Hilaire de la Celle de Poitiers le 27 août 1682, ${ }^{(53)}$ alors que le second a trait à une somme d'argent de "neuf cent francs à cent pistoles " subtilisée par "des Quidams [...] dans la Sacristie de l'Eglise du Bourg de Challans (diocèse de Luçon)" au début du mois de janvier $1779 .{ }^{(54)}$ Pour ce qui est du dernier acte de ce genre, c'est de loin le plus répréhensible puisqu'il est assimilé à un crime de "sacrilège » envers l'Église. Il concerne le vol de "deux Calices " et du "St Cyboire dans lequel ily avoit des Hosties consacrées " commis " en l'Église Paroissialle de Saint Cybard» de Poitiers "dans la nuit du seize au dix-sept " novembre 1714. ${ }^{(55)}$

Tout aussi graves, 5 autres monitoires dénoncent les atteintes aux prérogatives royales. Ces documents - qui concernent les fameux "cas royaux" définis par l'ordonnance criminelle de $1670^{(56)}$ - sont plus généraux que les précédents. Trois d'entre eux semblent ainsi avoir été publiés dans plusieurs régions de France : c'est le cas de l'appel à témoins daté du 30 octobre 1688 qui entend retrouver la trace des dangereux individus ayant troublé l'ordre public par des «assassinats, [des] vols [et des] pillages ${ }^{(57)}$ ", ou encore de ceux des 6 avril ${ }^{(58)}$ et 3 août $1716^{(59)}$ qui recherchent les quidams coupables de malversations commises au détriment des "finances de Sa Majesté ". Les deux derniers monitoires sont, en revanche, plus spécifiquement poitevins. Daté du 6 octobre 1702, le premier désire avoir des lumières sur les «frère \& sour demeurans depuis quelques années en cette ville de Poitiers [qui] auroient ramassé, billonné đo commercé les anciennes Espèces d'Or \& d'Argent, les ont réformées en fraude ou fait passer en les Provinces éloignées, \& même en les païs étrangers ${ }^{(60)}$ ". Quant au second, il a pour finalité de "découvrir ceux qui [...] ont gravé et fabriqué de faux timbres " en la "généralité du Poitou » au mois de juillet $1711 .^{(61)}$

Même si l'échantillon examiné n'est pas aussi important qu'on aurait pu le souhaiter, il livre malgré tout quelques résultats intéressants. En premier lieu, on remarque que la répartition des délits faisant l'objet de monitoires correspond - à peu de chose près - à celle des affaires jugées par les juridictions royales du siècle des Lumières : les histoires de vols y devancent largement les actes violents et les attentats à la religion et aux prérogatives royales. ${ }^{(62)}$ Toutefois, et à l'inverse, on note la sur-repré- 
sentation de deux catégories de "crimes " : les « divertissements » de biens chez les personnes défuntes (28\% du total), ainsi que les vols et les violences dont les victimes appartiennent aux catégories aisées de la population. Les "victimes » des actes dénoncés par ces 57 monitoires poitevins sont, en outre, très majoritairement issues de deux " milieux »: 21 d'entre elles (soit plus de $37 \%$ du corpus) sont effectivement des religieux ou des institutions cléricales, tandis que 12 autres (soit pratiquement $21 \%$ ) viennent au secours d'officiers ou de "services " royaux. Cela tendrait à prouver que l'on promulgue des monitoires dans le diocèse de Poitiers (et sans doute ailleurs, dans les autres régions de France) avant tout pour défendre les deux «piliers » du régime : l'Église catholique et l'administration du roi.

\section{Des avantages et des éventuels défauts}

Après avoir examiné la procédure et les affaires concernées, il convient enfin de s'interroger sur la réelle efficacité de ces appels à témoins. Donnent-ils des résultats intéressants, mais aussi quels sont leurs éventuels défauts ? Il faut savoir, en effet, que des critiques sont émises au siècle des Lumières au sujet de ces monitoires. Ces réserves proviennent aussi bien de rapports - très officiels - de l'Assemblée générale du clergé de France, ${ }^{(63)}$ que de textes et d'auteurs laïcs. ${ }^{(64)}$ C'est pourquoi il paraît important, à partir de notre échantillon, de répondre aux divers reproches que peut susciter ce genre d'action en justice entre 1670 et 1789 .

\section{Abuse-t-on de ce genre de procédure dans le diocèse de Poitiers?}

En premier lieu, on est en droit de se demander si cette procédure est fréquente, et si les juridictions de l'époque l'utilisent souvent. Le recours abusif à ces appels à témoignage est effectivement l'une des critiques que l'on rencontre dans quelques cahiers de doléances de $1789 .{ }^{(65)}$ Il reste à essayer de voir si c'est effectivement le cas dans le diocèse de Poitiers.

Pour la circonscription religieuse poitevine, il n'est pas possible de répondre - de manière précise - à cette interrogation, dans la mesure où les sources disponibles n'offrent pas l'opportunité de connaître la totalité des monitoires publiés entre 1670 et 1789 . En revanche, deux séries
1714, p. 128 ; J 1101

(monitoire du

22 novembre 1714).

(56) Marcel

MARION, Dictionnaire

des institutions de la France aux XVIİ̀me et XVIIIème siècles, Paris, 1923, p. 73.

(57) ADV, G 552, délibération capitulaire de Saint-Hilaire Le Grand de Poitiers du dimanche 31 octobre 1688, p. 63 (monitoire du 30 octobre 1688).

(58) ADV, J 1101 ; G 559, délibération capitulaire de Saint-Hilaire Le Grand de Poitiers du vendredi 10 avril 1716, p. 27 (monitoire du 6 avril 1716).

(59) ADV, G Supplément 6 (monitoire du 3 août 1716).

(60) ADV, J 1101 (monitoire du 6 octobre 1702).

(61) ADV, G 557, délibération capitulaire de Saint-Hilaire Le Grand de Poitiers du dimanche 19 juillet 1711 (monitoire du 11 juillet 1711). 
(62) Benoît GARNOT,

Crime et justice aux

XVIIème et XVIIIème

siècles, Paris, Imago,

2000, p. 12.

(63) DURAND DE

MAILLANE, $o p$. cit., t. IV, article "Monitoire", pp. 96-97.

(64) Raoul NAZ, article "Monitoire", dans Dictionnaire de droit canonique (sous la direction de R. NAZ), Paris, 1957, t. VI, colonne 942.

(65) Albert DESJAR-

DINS, Les cabiers des États Généraux en 1789 et la législation criminelle, Paris, 1883 ; René AUBIN, L'organisation judiciaire d'après les cabiers de doléances, Lille, 1962.

(66) Voir note 3.

(67) Alain CABAN-

TOUS, Les côtes barbares. Pilleurs d'épaves et sociétés littorales en France (16801830), Paris, 1993, p. 159. d'archives différentes permettent, malgré tout, d'avoir quelques éléments de réponse à ce problème. Les premiers documents à considérer sont, tout d'abord, les délibérations capitulaires de Saint-Hilaire Le Grand de Poitiers. ${ }^{(66)}$ A défaut d'être complets, ils mentionnent tous les appels à témoins publiés dans trois paroisses de la cité pictave (à savoir NotreDame de La Chandelière, Saint-Pierre l'Hospitalier et Sainte-Triaise) pour pas moins de 91 des 120 années de la période étudiée. Ils constituent donc un excellent échantillon (presque exhaustif) pour appréhender la fréquence des publications dans un quartier urbain du diocèse. Or, qu'observe-t-on dans ce secteur ? On note, d'une part, que les curés de paroisse sollicitent raisonnablement leurs ouailles pour ce genre d'action en justice : en l'espace de 91 ans, 38 monitoires seulement sont lus aux habitants du bourg de Saint-Hilaire, soit une moyenne de 4,17 monitoires par décennie. Quant à leur chronologie d'émission, elle s'avère très irrégulière sur l'ensemble de la période : ces appels judiciaires sont ainsi relativement nombreux jusqu'aux années 1720 (entre 9 et 13 par laps de temps de 20 ans), et rares - voire exceptionnels - entre 1730 et la Révolution. Les seconds documents à examiner sont, d'autre part, les cahiers de doléances poitevins pour savoir s'ils dénoncent - comme ceux du "pays" malouin ou des régions de Boulogne et de Calais ${ }^{(67)}$ - l'abus de cette pratique judiciaire dans la province. A vrai dire, leur lecture sur ce point est sans nuance : ni le clergé, ni la noblesse, ni le tiers état poitevin ne s'en soucient et n'en font écho dans leurs cahiers. On ne devait pas, par conséquent, ennuyer trop souvent les habitants du Poitou avec ces monitoires en 1789 , sinon ces derniers ne se seraient sans doute pas gênés pour en parler.

$\mathrm{Au}$ vu de ces quelques indices, on serait donc assez tenté de conclure au faible recours à ces appels à témoins dans le diocèse de Poitiers, surtout dans la seconde moitié du XVIIIème siècle.

\section{Ces monitoires sont-ils "efficaces" du point de vue judiciaire?}

L'autre problème que soulève l'usage de ces monitoires est celui de leur "efficacité" judiciaire. En effet, la publication de ces documents est-elle suivie d'effets, et permet-elle de résoudre certaines affaires qui s'enli- 
saient jusqu'alors ? La réponse à apporter à cette double interrogation n'est pas évidente, du moins à partir de notre petit échantillon. Elle ne peut être aussi que très nuancée, et même un peu contradictoire par certains aspects.

D’un côté, ces appels judiciaires n'entraînent visiblement pas souvent la réception de "révélations " par les curés de paroisse du diocèse de Poitiers. Ces documents, lorsqu'ils existent, se trouvent dans les minutes des juridictions d'Ancien Régime. Or, le moins que l'on puisse dire, c'est qu'ils semblent très peu nombreux dans les fonds d'archives poitevins. Les sondages effectués pour plusieurs justices du Poitou des XVIIème et XVIIIème siècles ne livrent effectivement que de très maigres résultats. ${ }^{(68)}$ Ainsi, on ne recense aucune "révélation " dans les dossiers du siège royal de Lusignan entre 1670 et 1700, ni d'ailleurs dans ceux des sénéchaussées de Civray entre 1760 et 1790 ou de Saint-Maixent entre 1780 et 1790 . On a la chance, en revanche, de découvrir deux petits recueils de dépositions dans les minutes de la justice seigneuriale de Saint-Hilaire Le Grand pour la période 1701-1788. Les archives du tribunal de la sénéchaussée et siège présidial de Poitiers sont toutefois un peu plus prolixes avec des " révélations » pour 7 affaires différentes : encore faut-il préciser que c'est au prix de dépouillements effectués sur près de 68 années d'activité judiciaire. Il n'empêche qu'on trouve, en fin de compte, très peu de dépositions consécutives à un monitoire dans les actes de justice poitevins. Cette très faible présence de "révélations " peut avoir plusieurs significations : soit les Poitevins concernés n’ont rien vu (cas le plus fréquent), soit ils éprouvent beaucoup de réticences à dire ce qu'ils savent sur telle ou telle affaire, soit ils ont peur des éventuelles représailles que pourraient provoquer leurs témoignages. Cela pourrait également vouloir dire que la menace d'excommunication n'a déjà qu'une très faible emprise sur les esprits de ce temps. En tout cas, quelle que soit la raison première à ce presque silence des habitants du diocèse, il semble révéler la très relative efficacité de ces appels à témoins.

Cependant, d'un autre côté, lorsque ces "révélations ${ }^{(69)}$ » existent, elles semblent considérablement faire avancer l'affaire. Sur les 9 dossiers pour lesquels on dispose de "révélations ", 8 au moins paraissent avoir été
(68) Voir note 5.

(69) Les 9 dossiers en question sont les suivants : ADV, G 663 (monitoire du 14 mars 1740) ; B I/2/58 (monitoire du 29 avril 1747) ; B I/2/67 (monitoire du 23 janvier 1750) ; G 663 (monitoire de mars ou avril 1750) ; B I/2/67 (monitoire du 13 juin 1750) ; B I/2/76 (monitoire du 1er juillet 1755) ; B I/2/89 (monitoire du 24 janvier 1759) ; B I/2/104 (monitoire du 17 janvier 1765) ; B I/2/180 bis (monitoire du 19 mars 1784). 
(70) ADV, B I/2/104, révélations de François de Lage datée du 27 janvier 1765, et de Jeanne Merceron datée du 29 janvier 1765 (consécutives au monitoire du 17 janvier 1765).

(71) ADV, B I/2/180 bis, voir notamment la révélation de Marie Anne Delage, datée du 17 avril 1784, faite auprès du curé de Lussac (consécutive au monitoire du 19 mars 1784).

(72) ADV, G 663, révélations recueillies par Barrault, curé de SainteTriaise de Poitiers, entre le 8 et le 25 mai 1750 (consécutives au monitoire sans doute daté de mars ou avril 1750).

(73) ADV, G 663, révélations recueillies respectivement par Guénard, vicaire de Sainte-Triaise (16 dépositions datées du 11 avril 1740),

Barrault, curé de SainteTriaise (59 dépositions sans date) et Billon, curé de Saint-Pierre L'Hospi- élucidés grâce aux dépositions recueillies par des desservants poitevins, suite à l'annonce d'un monitoire. Plus que le nombre, c'est d'ailleurs avant tout la précision et la qualité de ces témoignages qui offrent l'opportunité de retrouver les coupables. Ainsi, les deux uniques " révélations " transmises au mois de janvier 1765 à la "sénéchaussée et siège présidial de Poitiers " par le curé Guillemot permettent de découvrir les quidams qui "ont arraché dix-sept arbres " dans la propriété de Vincent Hilaire Du Tertre de la Coudre : il s'agirait en l'occurrence des dénommés René Marchand, Joseph Maltier et de quelques autres individus de la paroisse de Cissé. ${ }^{(70)}$ De même, les 6 Poitevins qui décident de dire à leur desservant ce qu'ils savent sur l'assassinat d'Antoine Micheau Dumeslier, subdélégué de l'intendant, confirment les autorités judiciaires dans leurs soupçons sur la personne du "sieur Chand de la Roderie » au mois d'avril 1784. ${ }^{(71)}$ Cependant, pour d'autres affaires, le nombre de paroissiens auditionnés par le responsable religieux est beaucoup plus important. Ils sont ainsi 40 à rendre visite au curé de SainteTriaise pour dénoncer ceux (il y en a plusieurs) qui auraient diverti des biens du domicile du défunt "marchand" Pierre Thoreau en $1750,{ }^{(72)}$ et même 80 à déposer auprès des desservants du bourg de Saint-Hilaire de Poitiers au sujet du vol perpétré chez le feu "chanoine" Raymond Sabourin de Marsilly en 1740 (la coupable est visiblement une certaine " dame de Vauvoux ${ }^{(73)}$ "). Cependant, le "record" en la matière appartient au dossier judiciaire relatif au monitoire du 1er juillet 1755 . Il est vrai que l'affaire est importante (la plus grave sans doute de notre corpus), et a alimenté les conversations durant plusieurs années. Elle concerne les actes de torture infligés à quatre huissiers de Châtellerault (à savoir les "nommés Moreau, Bachellier, Normand et Charpy ") par les hommes du marquis de Pleumartin le 8 mars 1753, suivis de l'exécution de deux brigadiers de la maréchaussée venus sur les lieux pour arrêter les coupables quelques jours plus tard (le 11 juin 1753). ${ }^{(74)}$ La justice a apparemment quelques difficultés à rassembler suffisamment de preuves accablantes contre le seigneur de cette petite bourgade (pourtant indéniablement responsable !), à tel point qu'elle décide de publier un monitoire plus de deux ans après les faits le 1er juillet 1755. En tout cas, même si elle 
paraît tardive, la procédure s'avère payante : pas moins de 203 habitants du secteur viennent alors dire ce qu'ils ont vu à leur curé, ce qui permet, en bout de course, aux autorités judiciaires de faire définitivement condamner le cruel marquis de Pleumartin. Par conséquent, ces quelques exemples tendraient à prouver la réelle "efficacité" de ces appels à témoins, du moins lorsqu'ils occasionnent des "révélations» de la part des Poitevins.

\section{Quels seraient les effets indirects de ces appels à témoins?}

Pour en terminer avec cet essai d'examen critique, on doit enfin s'interroger - à partir toujours de notre échantillon - sur les possibles travers de ce genre de procédure. En d'autres termes, quelles en sont les éventuelles imperfections directes ou indirectes?

A dire vrai, les dossiers judiciaires consécutifs à la publication d'un monitoire semblent révéler certains dysfonctionnements, voire quelques défauts. On est en droit, en premier lieu, d'émettre quelques critiques sur la date de promulgation de plusieurs d'entre eux. En effet, si l'on se fie à un corpus de 19 appels à témoins, leur publication a lieu parfois plusieurs mois après le délit incriminé. Certes, plus de la moitié (10 monitoires sur 19) sont prononcés dans les 30 jours qui suivent le crime. Ils sont cependant 9 (soit 47,38 \% du total) à être lus par les curés de paroisse après un délai d'au moins un mois, dont 2 de plus de six mois. Ces laps de temps relativement longs posent problème : les « révélations » recueillies alors reflètent-elles encore la réalité, et ne sont-elles pas plus des "reconstructions" raisonnées de la mémoire que de réels témoignages ? Au vu de la manière stéréotypée dont les quelques 186 habitants de Pleumartin relatent l'épisode du supplice des quatre huissiers de Châtellerault 28 mois après les faits, on peut légitimement se poser la question. ${ }^{(75)}$ En outre - ce serait la seconde observation à faire -, ces actions judiciaires semblent souvent alimenter les discussions dans les paroisses où elles sont promulguées. Cela se perçoit, dans les affaires pour lesquelles on possède des "révélations", à deux éléments. Tout d'abord, les «ouï-dire » et les paroles rapportées y sont très importants : ils représentent pas moins de 51 des 351 dépositions de talier (5 dépositions datées du 17 juillet 1740).

(74) ADV, B I/2/76

(monitoire du 1er juillet 1755), révélations recueillies respectivement par Mercier, curé de La Roche-Posay

(13 dépositions rédigées entre le 20 et le 26 juillet 1755), Roffay, curé de Châteauneuf de Châtellerault (4 dépositions rédigées entre le 18 et le 24 juillet 1755), et Desbordières, curé de Pleumartin (186 dépositions rédigées entre le 7 et le 26 juillet 1755).

(75) ADV, B I/2/76 (monitoire du 1er juillet 1755), révélations recueillies par Desbordières, curé de Pleumartin (186 dépositions rédigées entre le 7 et le 26 juillet 1755).

(76) ADV, B I/2/58 
(monitoire du 29 avril 1747), déposition de André, prieur curé de Saint-Martin d'Angles, du 1er juin 1747.

(77) ADV, B I/2/89 (monitoire du 24 janvier 1759), révélation de Radegonde Trouvé "domestique" faite auprès de Guéritault, curé de Saint-Jacques de Châtellerault, le 16 février 1759

(78) ADV, G 663, révélations de Marianne Baruteau et de Jean Pourvaceau auprès de Billon, curé de SaintPierre L'Hospitalier, datées du 17 juillet 1740 (monitoire du 14 mars 1740).

(79) ADV, B I/2/67, révélation d'Étienne Amillet recueillie par Bonnet, desservant de Saint-Porchaire de Poitiers, datée du 4 février 1750 (monitoire du 23 janvier 1750).

(80) DALLOZ, notre échantillon, soit presque $15 \%$ de l'ensemble. C'est le cas du témoignage de René de la Coste qui ne sait rien personnellement du dénombrement recherché par le monitoire du 29 avril 1747, mais qui a " ouy dire par [...] [le] curé de Saint-Pierre de Maillé qu'il croyait qu'il [...] [était] chez messire de la Toûche de la Guettière ${ }^{(76)}$ ". De même, la demoiselle Radegonde Trouvé de la paroisse Saint-Jacques de Châtellerault n'a pas d'information précise sur les « deux mille sept cent vingt une livres » volées en l'abbaye de l'Étoile au début du mois de janvier 1759, mais a cependant connaissance que le "bruit étoit que l'argent demandé par le monitoire seroit rendu $u^{(77)}$ ". Ces appels à témoins paraissent particulièrement propices au développement de rumeurs. Parfois, les choses vont même encore plus loin. Il arrive ainsi que ces monitoires soient à l'origine de menaces physiques et de tentatives d'intimidation. Certes, de tels faits sont rares, mais on a malgré tout repéré une situation de ce genre dans l'affaire du vol chez le défunt Emery Raymond Sabourin de Marsilly en 1740 : elle met en scène la dénommée "Madame de Vauxvoux " qui, surprise dans son méfait par "trois domestiques ", n'hésite pas à les menacer de "les faire tuer par son fils" s'ils " déclaroient quelque chose ". ${ }^{(78)}$ Enfin - et ce serait la dernière remarque à propos de cette pratique -, ces procédures judiciaires entraînent parfois la dénonciation de crimes autres que ceux du monitoire publié. Un tel résultat est sans doute inhabituel, mais n'est peut-être pas exceptionnel dans la mesure où l'on recense un cas de ce type dans notre petit échantillon. Il est le fait d'un certain Étienne Amillet qui, au lieu de donner des renseignements sur le vol perpétré chez « $M r D e$ Beauregard, [...] Subdélégué de Mr l'intendant » le soir de Noël 1749, révèle un autre délit, à savoir un second vol (de 400 livres tournois) commis par le frère de "François Mondon " au domicile du "nommé Lachattier ». (79)

En fin de compte, l'examen de ces quelques monitoires révèle certaines facettes intéressantes de la France d'Ancien Régime. Il permet, d'abord, de mieux cerner une pratique judiciaire à laquelle les Poitevins sont visiblement habitués entre 1670 et le commencement de la Révolution. Ainsi, les juridictions du diocèse l'utilisent souvent pour les affaires concernant des religieux ou des officiers royaux, les curés savent 
les promulguer, tandis que leurs ouailles ont une parfaite connaissance de ce qu'on attend d'eux en la circonstance. Certes, l'usage de ces appels à témoins présente quelques défauts et facilite l'expression de certains travers de la nature humaine (délations, tentatives d'intimidation, menaces...). Il semble cependant que l'on n'en abuse pas dans le diocèse de Poitiers, et que l'on y recourt beaucoup plus rarement dans la seconde moitié du siècle des Lumières. Quant aux « révélations» que ces actes peuvent susciter, leur intérêt dépasse le seul cadre de l'institutionnel et du judiciaire. Elles donnent effectivement à la fois des informations sur les mentalités des Poitevins (et leur éventuelle peur de l'excommunication), sur les liens existant entre les desservants et leurs administrés, et même sur l'état d'esprit et la sociabilité des hommes de ce temps. Bref, ce type de documents offre l'opportunité, pour l'historien, de mieux appréhender les sociétés paroissiales d'avant 1789. C'est sans doute le principal mérite de ces fameux monitoires, mal connus jusqu'à présent, et que la Révolution fait disparaittre définitivement dans les dernières années du XVIIIème siècle. ${ }^{\left({ }^{(0)}\right.}$
Répertoire méthodique et alphabétique de législation, de doctrine et de jurisprudence, Paris, 1853, t. XIV, article “Culte”, p. 757-758. 